\title{
PERSONALIDAD, TIPOS PATOGENOS EN ESTUDIANTES UNIVERSITARIOS
}

\author{
Luis A. Vicuña Peri. \\ Héctor Manuel Hernández Vals \\ Linda Marlene Pérez Álvarez. \\ Rune J. Arauco Mengoni
}

\begin{abstract}
RESUMEN
Los últimos 30 años de investigación psicológica nos permiten afirmar que muchas enfermedades físicas no tienen como etiología única a factores exógenos; gran parle de ellas se producen por el debilitamiento del sistema inmunológico, consecuencia de secuelas concomitantes del estado de estrés, como lo describen Chesney (1980), Eysenck (1987-1988), Grossarth-Malicek (1988) entre otros.

Esta investigación busca responder a la interrogante; ¿ Se encuentran diferencias significativas en función al sexo y a la elección profesional en una muestra de estudiantes universitarios de primer año en la UNMSM, de las Facultades de Psicología y Educación según los seis tipos de personalidad evaluadas con el Inventario de Reacciones Interpersonales de Eysenck y Grossarth-Maticek.

El método utilizado es el descriptivo comparativo. El test se aplicó en dos momentos con un intervalo de seis meses. Los resultados se interpretan en base a la permanencia en el mismo tipo en dos momentos diferentes o por el desarrollo favorable, esto es cuando en el segundo momento se presentan cambios favorables al sujeto. El desarrollo desfavorable es el proceso inverso.

En síntesis, en los estudiantes de psicología el tipo 1, 2 Y 5 representan el 39\% que presentarían riesgo de males oncológicos y coronarios. En educación el porcentaje de alumnos en riesgo llega al 30\%. La comparación porcentual no es significativa.

Finalmente el sexo sólo influye en el tipo 1 y en el tipo 5. En ambos casos los varones presentan una mayor predisposición a los males mencionados con respecto a las mujeres.
\end{abstract}

Palabras clave: Reacción interpersonal, Tipo / predisposición al cáncer; Tipo 2 predisposición a las enfermedades coronarias; tipo 3 histérico; Tipo 4 saludable; Tipo 5 racional-antiemocional; Tipo 6 psicopático.

\begin{abstract}
The last 30 years of psychological investigation they allow us to affirm that many physical illnesses don't have as unique etiologic to exogenous factors; great part of them takes place for the weakening of the immunology system, consequence of concomitant sequels of the stress stale, as Chesney describes it (1980), Eysenck (1987-1988), Grossarlh-Malicek (1988) among others.

This investigation looks for to respond to the query They are significant differences in function to the sex and the professional election in a sample of university students first years old in the UNMSM, of the Abilities of Psychology and Education according to the six types of personality evaluated with the Inventory of Interpersonal Reactions of Eysenck and GrossarthMaticek.

The used method is the descriptive one comparative. The test was applied in two moments with an interval of six months. The results are interpreted based on the permanency in the same type in two different moments 01' for the favourable development, this is when in the second moment favourable changes are presented to the fellow. The unfavourable development is the in verse process.

In synthesis, in the psychology students the type 1, 2 and 5 represent 39\% that would present risk of bad oncological and coronary. In education the percentage of students in risk arrives to $30 \%$. The percentage comparison is not significant.

Finally the sex only influences in the type 1 and in the type 5. In both cases the males present a bigger bias to the wrongs mentioned with regard to the women.
\end{abstract}

key Words: Interpersonal reaction, Type 1 bias to the cancer; Type 2 bias lo the coronary illnesses; Type 3 hysterical; Type 4 healthy; Type 5 antiemocional rational; Type 6 psychopathic. 


\section{INTRODUCCION}

Aunque la investigación acerca de la personalidad y diferencias personales desde comienzos del presente siglo ha identificado una plétora de rasgos que son útiles para estudiar y entender las diferencias individuales (extroversión, auto agresividad, necesidad de logros, etc.) muchos de estos rasgos están íntimamente relacionados a través del análisis estadístico y conceptual. Las investigaciones han tratado de reducir un gran número de razones superficiales y específicos a un grupo más pequeño y manejable de dimensiones subyacentes y fundamentales (Eysenck 1967, Cattell 1965).

A fines de los 50 dos cardiólogos Ray Rosenman y Meyer Friedman, acuñaron el término «Patrón de Conducta tipo A» para referirse a una constelación de señales, signos de comportamiento evidentes, manifiestos, latentes y disposiciones de personalidad subyacentes que ellos creyeron colocaban al individuo en un mayor riesgo de desarrollar enfermedades del corazón.

La dicotomía tipo A - B es ahora bien conocida y hay cierta evidencia que la une a las enfermedades o afecciones a la arteria coronaria (Friedman y Rosenman,1974).

De manera similar para el cáncer parece existir un Tipo C el cual caracteriza a los pacientes de cáncer y pronostica la enfermedad (Baltrusch, Stangel y Waltz, 1988; Eysenck 1985).

Varios estudios prospectivos han demostrado recientemente la predictibilidad del cáncer y de las enfermedades coronarias sobre un periodo de entre 10 y 20 años. Sobre estos descubrimientos Eysenck y Grossarth-Maticek elaboraron el Inventario de reacciones interpersonales donde el stress juega un papel importante, pues existe ahora evidencias que la personalidad y el stress están relacionados causalmente con varios tipos de enfermedades incluyendo el cáncer y enfermedades al corazón.

Desde esta perspectiva creemos que será de utilidad explorar los tipos de personalidad en dos grupos de estudiantes de la Universidad Nacional Mayor de San Marcos unos pertenecientes a la Facultad de Psicología y el otro a la Facultad de Educación y despejar la siguiente interrogante:

¿Se encuentran diferencias significativas en función al sexo y a la elección profesional en una muestra de estudiantes que cursan el primer año de estudios universitarios según los seis tipos de personalidad evaluadas con el Inventario reacciones interpersonales de Eysenck y Grossarth-Maticek?

Son los objetivos del presente estudio:

a) Identificar si la pertenencia a un campo de profesionalización y el sexo influye sobre los tipos de personalidad y con ello a cierta premorbidad.

b) Identificar los tipos de personalidad de mayor porcentaje enfatizando en aquellos, que están asociados a la premorbidad hacia males oncológicos y cardiovasculares.

c) Sobre las conclusiones se podrán elaborar programas orientados a menguar los rasgos de personalidad de alta premorbidad.

\section{Importancia del estudio}

Siendo la salud un problema social complejo que rebasa bastante el campo de la atención médica, requiriendo de acciones multidisciplinarias; el presente estudio de enfoque psicológico es de carácter preventivo orientado a la detección de tipos de personalidad en futuros profesionales que podrían verse frustrados en su realización por el desarrollo de males físicos facilitados a partir del tipo de personalidad, más aún cuan-do sabemos que en el Perú, en 1988, más de uno de cada seis defunciones generales se debieron a 
enfermedades respiratorias agudas, el segundo lugar de causa de mortalidad está representado por el grupo de las enfermedades cardiovasculares en el 14,02\%, el tercer lugar trascendente con casi un $8,44 \%$ las muertes por diferentes cuadros oncológicos. Las afecciones inmunoprevenibles ocupan el cuarto lugar con un $7.10 \%$, el 7,08\% está representado por afecciones originadas en el periodo perinatal. Los traumatismos y envenenamientos ocupan el sexto lugar con el $6,84 \%$, las muertes por enfermedades infecciosas intestinales alcanza al $6.32 \%$ y por deficiencias en la nutrición se tiene el 2,91 $\%$ de los fallecimientos según fuente D.T.T.D del Ministerio de Salud 1988. Como se puede observar el $22.46 \%$ de los fallecimientos en el Perú está, asociados a males cardiovasculares y oncológicos, razón suficiente para asumir con preocupación el estudio de factores de premorbidad en el desarrollo de estas enfermedades que según lo expuesto líneas arriba están asociadas con algunos tipos de personalidad y por ende, el diagnóstico y la intervención oportuna pueden incidir positivamente sobre la prevención.

\section{MARCO TEÓRICO}

\section{INVESTIGACIONES PREVIAS}

Chesney, (1980): Se encuentra cierta evidencia que los une a las enfermedades coronarias y la personalidad de tipo A-B. Estos resultados son empañados por las dificultades para determinar el tipo A, caracterizado porque sólo existe acuerdo en torno a ciertos rasgos dentro del grupo A, tales como hostilidad y agresión.

Eysenck (1988): Reporta que hay relación entre la propensión al cáncer y rasgos como ser exageradamente cooperadores, pacificadores, tímidos, sumamente pacientes y que evitan los conflictos, con la propensión al cáncer e impotentes frente al estrés interpersonal, lo que conlleva a sentimientos de desesperanza e inutilidad y finalmente depresión, lo que lleva a niveles altos de cortisol y su secuela de deficiencias inmunológicas (Gossarth-Maticek y Eysenck, 1990).

Eysenck, (1987a, 1987b, 1987c, 1988a, 1988b); Grossarth-Maticek, Eysenck y Vetter (1988); Grossarth-Maticek, Eysenck, Vetter y Frentzel-Beyme (1988) demuestran la predictibilidad del cáncer y de las enfermedades coronarias en períodos de 10 Y 20 años. Se utilizaron entrevistas y cuestionarios a varios tipos, basados en ciertas teorías concernientes a la estructura de la personalidad y las conductas de las personas con propensión al cáncer y a enfermedades de la coronaria. Pese al éxito alcanzado se optó por mejorar las propiedades psicométricas de los instrumentos utilizados e incrementar su predictividad mediante ciertos cambios.

Baltrush, Stangeli, Waltz (1988); BoothKewly y Friedman (1987); Eysenck (1984, 1985, 1988b, 1990, 1991); Eysenck y Eysenk (1985); Friedman y Booth-Kewly (1987); Grossarth-Maticek, Eysenck, Vetter y Frentzel-Beyme (1986); Roseman y Shesney (1980); Taylor (1984); Thomas (1988); y Whitin (1965) encuentran evidencias de que la personalidad está relacionada con varios padecimientos y enfermedades psicosomáticas, así como el estrés y el enfrentamiento a problemas.

\section{RELACION DEL TIPO DE PERSONALIDAD CON COMPONENTES ESPECIFI- COS DE LA PERSONALIDAD}

\section{Tipos de personalidad y ansiedad}

Scmitz (1991) encuentra correlaciones positivas entre los tipos 1 y II, Y también el tipo cuatro con la Ansiedad de Estado medida con la escala de Endler, provenientes de las EMAS (Endler, Edwards y Vitelli, 1990a). Disgregando la Ansiedad de Estado en sus componentes «emocionales autonómicos» $\mathrm{y}$ «de preocupación-cognitivos», se descubrió 
que, a excepción del tipo 1, sólo el componente «preocupación-cognitivo» se encuentra marcadamente involucrado en el patrón de conducta de los tipos I, II, III y VI.

\section{Tipos de personalidad y dogmatismo}

Scmitz también evaluó la dimensión «mentalidad cerrada» o «dogmatismo» (Rokeach, 1960). Rokeach sostiene que la «mentalidad cerrada» muestra alguna afinidad con la rigidez, pero está menos marcada en situaciones en que una persona tiene que romper un campo existente que en aquellas situaciones en que se tiene que integrar nuevas creencias al sistema de conducta existente.

Como resultado se encontró que cada tipo, con excepción del tipo 4, se encuentra correlacionado positivamente con el puntaje total de la mentalidad cerrada o dogmatismo. Un patrón similar fue observado para los componentes más cognitivos de! dogmatismo, las dimensiones «Creencia en una Verdad»y «Creencia en una Causa».

Estos cinco tipos y en especial 1,2 y 3, pueden ser caracterizados como fijados emocionalmente en alto grado y dependientes emocionalmente, poco flexibles en su conducta. Estos hallazgos concuerdan con e! descubrimiento de que los tipos 1, 2 y 3, a diferencia del tipo 4, muestra evidencia de prejuicio en una manera muy marcada (GrossarthMaticek, Eysenck y Vetter, 1969). Si se asume como válida la afirmación de Rokeach de que la «mentalidad cerrada» sirve a la necesidad de prevenir mecanismos amenazadores de la realidad» y que un sistema cerrado es «nada más que la red total del mecanismo de defensa psicoanalítica y las correlaciones positivas entre el puntaje total de dogmatismo con el neurotismo y la introversión (Eysenck, 1956 y Schmitz, 1985). La conclusión a la que se puede llegar es que los tipos de conducta se correlacionará con ciertas clases de defensas y estrategias de enfrentamiento de problemas.

\section{Tipos de personalidad y enfrentamiento de problemas}

Otra relación importante es la que se puede establecer con los patrones de enfrentamiento de problemas, motivo por el cual se determinó el carácter de la vinculación del tipo de personalidad con la Alexitimia y los resultados del Inventario de Problemas de Endler para situaciones de bastante estrés (CISS) (Endler y Parker, 1990).

Sobre estos hallazgos se esperaba que de todos los tipos de personalidad, los tipos 1 y 2 se correlacionarían positivamente con la alexitimia y se encuentra que las personas clasificadas en estos tipos presentan dificultades para describir y comunicar sus sentimientos reales. El tipo 5, al que se supone predispuesto a la depresión endógena, tiene dificultad para comunicar sus sentimientos y el tipo 6 , al que se supone psicopático, tiene dificultades para la descripción de sentimientos.

Como resultado de la aplicación del CISS (escala de enfrentamiento de problemas, desarrollada por Endler y Parker; 1990) se descubrió que las personas del tipo 4, las personas saludables, se encuentran positivamente relacionadas con la conducta «orientada a tareas», mientras que los tipos 1, 2 y 3 se encuentran correlacionados positivamente con la conducta «orientada a la emoción» y «orientada a la evasión». El tipo 5 (el psicópata) muestra una correlación negativa con la conducta «orientada a tareas» y una correlación positiva con la conducta «orienta da a la emoción». Este hallazgo parece tener sentido debido a que los psicópatas tienen con frecuencia poco interés por una tarea constructiva y con frecuencia son muy impulsivos en situaciones de estrés.

En 1985, Taylor propuso el constructo hipotético de alexitimia, derivándolo de observaciones clínicas de pacientes con enfermedades psicosomáticas clásicas. Las personas alexi-tímicas se caracterizan por ser personas que tienen dificultades en identificar y describir sus sentimientos, su estilo cognitivo es concreto y basado en la realidad. Sus relaciones interpersonales pueden clasificarse como simbióticas, mostrando poca autonomía. 
Un alto grado de alexitimia está relacionado con la colitis ulcérica (Jackson, 1977), pancreatitis crónica (Nakal, Sugita, Nakagawa, Araki e Ikemi, 1974) asma (Kleiger y Jones, 1980), enfermedades cardiocoronarias (Dongler, 1974), etc.

\section{Relaciones entre tipos de personalidad y formas de reacción}

Otro punto de interés fueron las formas de reacción frente a situaciones percibidas por el sujeto como de bastante estrés.

Con tal propósito se aplicó el método de Thomas por el cual el sujeto disponía de una variedad de formas diferentes de reacción que fueron organizadas en un orden jerárquico. Una persona fue asignada a un cierto tipo de conducta en el caso de que él cayera en el cuartil de la escala de un tipo de conducta. El hallazgo más notable es que a pesar de las grandes diferencias inter e intra individuales, ciertas formas de reacción son encontradas más frecuentemente en un único tipo de conducta que en otro, como se puede observar en el cuadro No. 1.

\section{Cuadro No.1}

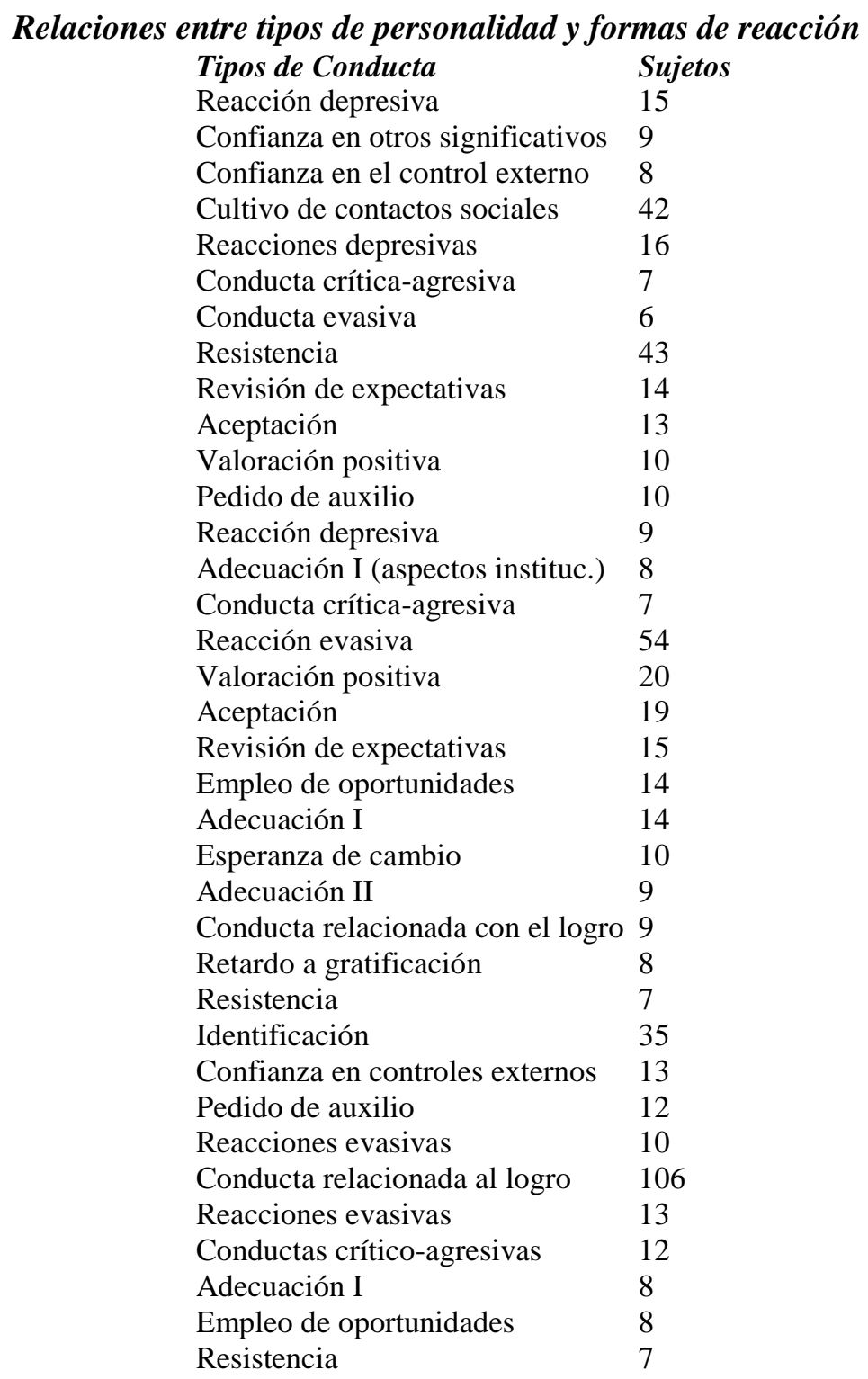

Asignación múltiple de reacciones al estrés reportadas a varias categorías de formas de reacción para personas que pertenecen a uno de los 6 tipos de conducta presentados. 


\section{Personalidad, quejas y enfermedades psicosomáticas}

La investigación de Schmitz, 1990, presenta también algunos hallazgos relacionados con la vinculación entre «tipos de conducta» y las «quejas psicosomáticas».

Las escalas psicosomáticas fueron evaluadas mediante Escalas de Clasificación, encontrándose un patrón correlacional similar para tipos 1 y 2, los que están relacionados positivamente con los índices para las tendencias depresivas, cansancio físico, enfermedades cardiovasculares, incapacidad para separarse, insomnios; y uso y abuso de drogas psicofárma-cológicas, alcohol, narcóticos y el consumo de tabaco.

En suma en la presente investigación no se persigue desarrollar una tipología de la personalidad que resultaría «rígida» o «limitan te» más bien compartimos el interés por el estudio del individuo dentro de determinada facetas psicológicas, como los procesos de interacción social, la expresión de la emocionalidad o las propias manifestaciones comporta-mentales; de cada una de estas facetas.

Muchos investigadores han buscado identificar aspectos específicos de los patrones de conducta que pueden ser particularmente patógenos.

Hay evidencias que la personalidad y el estrés están relacionados causalmente con varios tipos de enfermedades incluyendo el cáncer y enfermedades al corazón. Los datos que se disponen ahora son suficientes para indicar que es así.

Es a partir de estas inquietudes teóricas que desarrollamos el presente estudio.

\section{DEFINICION DE CONCEPTOS PERSONALIDAD}

La perspectiva teórica que resulta utilizada es de corte psicosomático debido a que es imposible atribuir exclusivamente a factores orgánicos el desencadenamiento de problemas específicos de salud, encontrándose que los individuos con personalidad considerada como sana son menos propensos a contraer los males que se intenta predecir. Se llega a esta conclusión luego de establecer la relación entre conjuntos de rasgos que caracterizan cada uno de 6 tipos de personalidad rotulados con números que van del 1 al 6 .

a) Son reacciones a situaciones llenas de estrés y según la muestra de investigación (Grossarth-Maticek; 1986 y 1988) se encuentran parcialmente influenciadas por las experiencias de la vida del individuo y por tanto pueden ser modificadas mediante intervenciones terapéuticas.

b) Se puede suponer que estos tipos, al igual que las otras clases de conducta social (Eysenck y Eysenck, 1985) se encuentran también influenciadas por las dimensiones básicas de la personalidad, tales como el Neurotismo (N), Extraversión (E) y Psicotismo (P). En 1987 Schmitz descubrió relaciones significativas entre N, E y la Escala de Mentira (L) por un lado y los 4 primeros tipos, quedando pendiente la relación entre $\mathrm{P}$ y los tipos 5 y 6.

Estos tipos de conducta o de personalidad presentan las siguientes peculiaridades:

Tipo 1: Predisposición al Cáncer: Definido mediante una dependencia conformista frente a la renuncia al objeto. Son personas que persiguen intensamente metas altamente valoradas (persona, objeto, situación) las cuales desafortunadamente se encuentran inaccesibles para él o ella. Son altamente dependientes de otros, rígidos, con tendencias depresivas, se inhiben de mostrar o comunicar sus sentimientos.

Tipo 2: Predisposición a la Enfermedad coronaria: Es definido mediante la dependencia conformista a un objeto de molestia. Una persona de este tipo trata inútilmente de escapar y de emanciparse de una persona o un objeto que le es emocionalmente muy importante, su conducta es también muy rígida y a menudo depresiva. Al igual que el tipo 1, le es difícil mostrar sentimientos de ira y fastidio.

Tipo 3: Tipo Histérico. Es descrito mediante una dependencia no conformista a un objeto que es tanto de renuncia y molestia. Sus características suelen alternar entre las dos formas 
generales de reacción de los tipos 1 y 2. Resulta improbable que mueran de cáncer o de enfermedad coronaria. Está protegido hasta cierto grado.

Tipo 4: Tipo Saludable. Está caracterizado por la autonomía.

Tipo 5: Tipo Racional-Antiemocional. Presenta tendencia a emitir reacciones racionales y antiemocionales. Se predice como propenso a la depresión endógena y al cáncer.

Tipo 6: Tipo Psicopático. Se caracteriza por conductas claramente antisociales y posiblemente criminales. Son personas propensas a la adicción a drogas.

\section{ESTANCAMIENTO.}

Se denomina a la retención o incremento del puntaje que lo establece como propenso a ciertas enfermedades. El estancamiento conduce a un pronóstico desfavorable. Es aplicado a los tipos 1,2 y 5 .

\section{DESARROLLO DESFAVORABLE:}

Se denomina así a la reducción del puntaje que indica propensión a una enfermedad o el incremento de los tipos saludables o relativamente favorables. Se aplica a la reducción en 1 , 2 y 5 y el aumento para 3,4 Y 5 .

\section{METODOLOGIA}

El método seguido para despejar el problema formulado es descriptivo comparativo, en cuanto queremos averiguar si en función a la elección profesional y sexo hay predominio significativo de alguno de los seis tipos de personalidad inventariados, así también identificar aquellos tipos asociados a la premorbidad oncológica, cardiovascular, propensión a la drogadicción y criminalidad.

La muestra ha sido elegida por el método accidental no probabilística debido principalmente a que no todos los estudiantes mostraron interés. La muestra original estuvo constituida por 300 estudiantes 200 de la Facultad de Educación y 100 de la Facultad de Psicología, la misma que se vio reducida a 141 de los cuales 72 pertenecen a Educación y 69 a Psicología, esta reducción se debió que para efectos del estudio se requiere de dos aplicaciones con un intervalo de seis meses, hecho que dificultó la localización de los examinados para la segunda aplicación. Distribución que a continuación se reproduce.

\section{Cuadro No. 2}

Distribución muestral en función a la Facultad de pertenencia y sexo.

\begin{tabular}{|l|c|c|c|}
\hline $\begin{array}{c}\text { Facultad } \\
\text { Sexo }\end{array}$ & Psicología & Educación & Total \\
\hline Varón & 30 & 37 & 67 \\
Mujer & 39 & 35 & 74 \\
\hline TOTAL & $\mathbf{6 9}$ & $\mathbf{7 2}$ & $\mathbf{1 4 1}$ \\
\hline
\end{tabular}

El instrumento utilizado fue el «Inventario de Reacciones Interpersonales» de Eysenck y Grossarth-Maticek. Este inventario es la versión corta del short interpersonal reactions inventory (SIRI) (Grossarth-Maticek y Eysenck, 1990), (inventario de reacciones interpersonales: Chorot y Sandin, 1991:

Sandin y Chorot, 1992) Representa la forma psicométrica más refinada y abreviada, formulada por estos autores para evaluar los tipos de reacción al estrés psicosocial (es conveniente recordar que Eysenck y GrossarthMaticek habían estado empleando cuestionarios $\mathrm{y}$ entrevistas de diferentes tipos y de niveles de fiabilidad y validez relativamente heterogéneos).

Tanto este inventario como una versión excesivamente larga (el Personality Stress 
Questionaire) constituyen una importante mejora en comparación con las anteriores versiones, respecto a sus propiedades psicométricas y predictivas.

El inventario consta de 70 items de 2 alternativas (SI-NO). Puede administrarse tanto de forma individual como colectiva. El examinado debe contestar a cada item con un «SI» 0 un «NO». Permite evaluar los seis tipos definidos por Eysenck y GrossarthMaticek.

El SIRI incluye dos formas de evaluación del tipo 4. Una mediante items formulados de manera positiva (tipo 4a) y una segunda a través de preguntas formuladas de manera negativa para controlar la aquiescencia (tipo 4b). Eysenck y Grossarth-Maticek asumen que desde el punto de vista psicosomático los tipos 3,4 y 6 pueden ser considerados globalmente como relativamente saludables, en oposición a los tipos 1, 2 y 5, que serían insanos, al menos en base en los trabajos psicométricos llevados a cabo por dichos autores.

Para la confiabilidad y validez del instrumento se aplicó la prueba a sujetos normales (no clínicos), estudiantes de primer ciclo universitario (160 mujeres y 140 varones), con un rango de edad entre 18 y 25 años. Se aplicó colectivamente la versión española del Short Interpersonal Reactions Inventory (Grossarth-Maticek y Eysenck, 1990, Inventario de Reacciones Interpersonales: Chorot y Sandín, 1991; Chorot, Sandín y Navas, 1991; Sandin y Chorot, 1992). Los sujetos contestaron la prueba de 70 items. Todos recibieron las mismas instrucciones $\mathrm{y}$ fueron motivados de forma semejante, participando voluntariamente en la investigación. Además del SIRI, los sujetos cumplimentaron el Inventario de Auto evaluación STAXI (Spealberger, 1988) y la Escala de Síntomas Somáticos (ESS) de Sandín y Chorot (1987).

La validez fue de constructo mediante el análisis factorial y la confiabilidad por la consistencia interna mediante la ecuación alfa para cada una de las 6 escalas (tipos) del cuestionario SIRI así como también los resultados de los análisis factoriales que se reproducen en el siguiente cuadro: Respecto a los coeficientes alfa estos tienden a situarse por encima del valor de 0,70 con la excepción de la escala 5 que no alcanza el 0,60. Los análisis factoriales revelan la existencia de dos factores tanto en varones como mujeres, a la vez que una configuración factorial equivalente en ambos grupos. El primer factor parece estar configurado básicamente por los tipos 1 y 2, mientras que el segundo factor está más determinado por los tipos 3 y 6 , como podrá verse en el siguiente cuadro.

Cuadro No. 3

Análisis Factorial (Baremax) para varones y mujeres (nos indica los pesos factoriales inferiores a +/- 0,20 Y coeficientes alfa relativos a las subescalas del SIRI obtenido por Grossarth-Maticek y Eysenck.

\begin{tabular}{|c|ccl|ccc|}
\hline \multicolumn{5}{|c|}{ VARONES } & \multicolumn{3}{c|}{ MUJERES } \\
\hline $\mathbf{T}$ & F1 & F2 & r20 & F1 & F2 & r20 \\
\hline $\mathbf{1}$ & 0.60 & - & 0.70 & 0.79 & - & 0.77. \\
$\mathbf{2}$ & 0.77 & -.20 & 0.66 & 0.75 & 0.37 & 0.82 \\
$\mathbf{3}$ & 0.47 & 0.28 & 0.68 & 0.35 & 0.74 & 0.71 \\
$\mathbf{4}$ & -.85 & - & 0.81 & -.87 & -.31 & 0.84 \\
$\mathbf{5}$ & - & 0.22 & 0.53 & - & 0.38 & 0.58 \\
$\mathbf{6}$ & 0.65 & 0.60 & 0.70 & 0.20 & 0.71 & 0.65 \\
\hline
\end{tabular}

Varianza Explicada: $67 \%$ Varianza Explicada: $66 \%$

En relación con la fiabilidad del inventario en términos generales los coeficientes alfa son bastante elevados, con la pequeña excepción de la subescala correspondiente al tipo 5, cuyo coeficiente alfa no supera al 0.60 , tanto en mujeres como en varones. 
La consistencia interna de la escala, tal y como se refleja a través del análisis factorial es congruente con los datos factoriales obtenidos por los autores del cuestionario, por ejemplo, confirmación de un factor asociado a los tipos 1 y 2 (riesgo somático) y un segundo factor más, determinado por los tipos 3 y 6 (histeria/psicopatía). Por otra parte parece razonable que los pesos factoriales del tipo 4 (tipo saludable) sean de valencia negativa en ambos factores.

La validez del cuestionario convergente-divergente y predictiva se ha analizado mediante sus correlaciones con el STAXI y la ESS, respectivamente. Los datos sobre la validez predictiva ponen de manifiesto:

1. Un cierto grado de especificidad entre el tipo 2 Y los síntomas cardiovasculares, respiratorios y gastrointestinales;

2. Una mayor vinculación del tipo 1 con el sistema inmunológico (esta subescala, no obstante no mide cáncer exclusivamente, sino manifestaciones inmunológicas más generales, $y$

3. Una relación negativa entre el tipo 4 y las distintas manifestaciones somáticas.

En el presente estudio se revisó la fiabilidad por el coeficiente alfa, con una muestra de 141 estudiantes de primer año pertenecientes a la Facultad de Educación y Psicología que a continuación reproducimos:

\begin{tabular}{|c|c|}
\hline TIPO & r20 \\
\hline 1 & 0.82 \\
2 & 0.85 \\
3 & 0.76 \\
$4 \mathrm{a}$ & 0.73 \\
$4 \mathrm{~b}$ & 0.83 \\
5 & 0.80 \\
6 & 0.78 \\
\hline
\end{tabular}

A diferencia de lo hallado por los autores en todas las subescalas encontramos coeficientes de fiabilidad mayores de 0.70 .

Es conveniente señalar que el inventario de reacciones interpersonales fue traducida directamente del inglés utilizando el método de doble traducción, para obtener el control idiomático. Luego se aplicó a un grupo piloto con el propósito de detectar vocablos con denotaciones diferentes a las esperadas de cuyo resultado se mejoraron los Ítems. Luego fueron aplicados a la muestra de estudio sin reportar dificultad de comprensión.

La interpretación de cada una de las subescalas está basada en los cuartiles de los puntajes directos de modo que los sujetos que obtengan un puntaje igualo mayor que siete en cualquiera de las escalas significa que presenta un tipo definido según la escala.

Los sujetos que puntúan de 3 a menos están comprendidos en el cuartil 1, que indica rasgos irrelevantes del tipo que la escala mide.

Las puntuaciones entre los rangos 4 y 6 ubican al sujeto en el cuartil 2, de característica ambivalentes según la escala.

\section{Recolección de Información}

El inventario se aplicó en grupos no mayores de 25 examinados por vez, impartiéndoles las mismas instrucciones e información motivadora para participar en el estudio.

\section{Método Estadístico}

Para averiguar si la Facultad de pertenencia y el sexo tienen alguna influencia en los tipos 
de personalidad se ha utilizado el análisis de varianza. Para determinar la permanencia o conservación, el cambio favorable, el cambio desfavorable y la no pertenencia a un tipo de personalidad se utilizó el análisis de contingencia de Mac Nemar, donde cada examinado es su propio control, del momento 1 a un intervalo de 6 meses al momento 2.

\section{Análisis de Resultados}

Con el propósito de despejar la interrogante formulada respecto a si los tipos de personalidad difieren en función a la facultad de pertenencia y el sexo, los resultados del Inventario de Reacciones Interpersonales fueron procesados mediante el análisis de varianza para dos factores (Facultad de pertenecía y sexo) y cada uno en dos dimensiones (Educación y Psicología; Varones y Mujeres).

Como el Inventario de Reacciones Interpersonales, para establecer el tipo premórbido y saludable de personalidad requiere de dos aplicaciones con un intervalo de seis meses, los resultados adicional-mente fueron analizados en función a: la conservación, desarrollo positivo y negativo; con estos datos se procedió al análisis de contingencia donde cada sujeto es su propio control utilizando para ello la ecuación de Mac Nemar.

Finalmente se hace una descripción porcentual de la evolución de las reacciones interpersonales, tanto de la premorbidad como de la personalidad saludable.

Datos que a continuación se presentan en el mismo orden en que se ha señalado.

Las reacciones interpersonales en función a la facultad de pertenencia y el sexo.

Para facilitar la interpretación en primer lugar hemos creído conveniente reproducir las medias aritméticas del Inventario de Reacciones Interpersonales, obtenidas por cada grupo de contrastación y pertenecientes a los dos momentos del examen que se consignan en el siguiente cuadro:

Cuadro No. 4

Medias Aritméticas correspondientes a los varones y mujeres de la Facultad de Psicología y Educación en función al tipo de Personalidad en el MO1 y MO2.

\begin{tabular}{|c|lc|cc|cc|cc|}
\cline { 2 - 9 } \multicolumn{1}{c|}{} & \multicolumn{4}{c|}{ EDUCACIÓN } & \multicolumn{3}{c|}{ PSICOLOGÍA } \\
\cline { 2 - 10 } \multicolumn{1}{c|}{} & \multicolumn{3}{c|}{ V } & \multicolumn{3}{c|}{ M } & \multicolumn{3}{c|}{ V } & \multicolumn{3}{c|}{ M } \\
\hline TIPO & MO1 & M02 & MO1 & M02 & MO1 & M02 & MO1 & M02 \\
\hline $\mathbf{1}$ & 4.84 & 4.00 & 4.14 & 3.54 & 5.03 & 4.57 & 3.64 & 3.28 \\
$\mathbf{2}$ & 3.84 & 3.54 & 4.00 & 3.86 & 3.70 & 2.97 & 2.87 & 2.46 \\
$\mathbf{3}$ & 4.16 & 3.95 & 4.23 & 3.46 & 4.24 & 3.71 & 3.15 & 3.32 \\
$\mathbf{4}$ & 7.00 & 7.14 & 6.74 & 6.60 & 6.93 & 7.03 & 649 & 6.72 \\
$\mathbf{5}$ & 2.68 & 2.59 & 2.53 & 2.09 & 3.20 & 2.48 & 213 & 2.23 \\
$\mathbf{6}$ & 3.14 & 3.16 & 3.6 & 3.14 & 3.43 & 2.70 & 246 & 2.18 \\
\hline
\end{tabular}

En una visión panorámica se observa que en los varones de ambas Facultades hay tendencia a la premorbidad oncológica (Tipo 1); en los estudiantes de ambos sexos y ambas Facultades los promedios se mantienen con tendencia a la ausencia de premorbidad cardiovascular (Tipo 2); los promedios hallados en el Tipo 3 deja entrever tendencia a los bajos rasgos histéricos, que según los autores los sujetos definidos en este tipo estarían protegidos contra males oncológicos y cardiovasculares; por los promedios registrados en el Tipo 4 la tendencia de los estudiantes de ambos sexos y Facultades se orientan hacia la personalidad saludable; en el Tipo 5 los promedios relativamente bajos dejan entrever una tendencia a no apelar al control emocional mediante la racionalización, hecho que resulta positivo; finalmente por los promedios registrados en el Tipo 6 que evalúa rasgos psicopáticos con tendencia a la drogadicción y criminalidad se encuentran dentro de los límites del cuartil uno, por ende no hay mayores problemas al respecto.

En el siguiente análisis veremos si las características señaladas panorámicamente están sujetas a influencias para lo cual sometimos los resultados a la razón F de Fisher, para los 
dos momentos de la evaluación cuyos resultados se reproducen a continuación:

\section{Cuadro $\mathrm{N}^{\circ} .5$}

Resumen de Análisis de Varianza (Razón $F$ de Fisher) de los tipos de personalidad en función a la Facultad de Pertenencia, Sexo e Interacción

\begin{tabular}{|l|c|c|c|c|}
\hline \multirow{2}{*}{ TIPO: VARIABLES } & \multicolumn{2}{|c|}{ 1ra. Aplicación } & \multicolumn{2}{c|}{ 2da. Aplicación } \\
\cline { 2 - 5 } & F & Desic. & F & Desic. \\
\hline Tipo 1 por Facultad & 0.53 & NS & 0.03 & NS \\
Tipo 1 por Sexo & 8.29 & S & 5.22 & S $>$ Homb \\
Tipo 1 Interacción & 17.26 & S & 11.77 & S \\
\hline Tipo 2 por Facultad & 1.42 & NS & 7.18 & S>Educ \\
Tipo 2 por Sexo & 0.10 & NS & 0.18 & NS \\
Tipo 2 Interacción & 3.82 & NS & 1.40 & NS \\
\hline Tipo 3 por Facultad & 3.00 & NS & 0.45 & NS \\
Tipo 3 por Sexo & 2.57 & NS & 1.94 & NS \\
Tipo 3 Interacción & 7.66 & S & 3.74 & NS \\
\hline Tipo 4 por Facultad & 0.39 & NS & 0.01 & NS \\
Tipo 4 por Sexo & 1.35 & NS & 1.96 & NS \\
Tipo 4 Interacción & 2.70 & NS & 4.05 & S \\
\hline Tipo 5 por Facultad & 0.01 & NS & \multirow{2}{*}{0.00} & NS \\
Tipo 5 por Sexo & 3.25 & NS & 1.46 & NS \\
Tipo 5 Interacción & 8.80 & S & 3.09 & NS \\
\hline Tipo 6 por Facultad & 1.88 & NS & 4.71 & S>Educ \\
Tipo 6 por Sexo & 0.59 & NS & 0.86 & NS \\
Tipo 6 Interacción & 5.25 & S & 1.97 & NS \\
\hline \multicolumn{2}{|c}{$g l-137$} & $F^{\prime} a l$ & $0.05=3.92$ & \\
\hline
\end{tabular}

De la lectura del cuadro se desprende:

a) En el Tipo 1, la Facultad de Pertenencia no agrupa significativamente a estudiantes con premorbidad oncológica; pero sí encontramos diferencia significativa al 0,05 de margen de error en función al sexo siendo los varones quienes tienden a ubicarse con un mayor número de rasgos correspondientes a este Tipo; y al resultar la interacción significativa, ratifica que al margen de la opción profesional elegida es el sexo, en este caso los varones; lo que delimita la mayor incidencia.

b) En el Tipo 2, resulta importante destacar que en el momento 2 (luego de seis meses de la primera evaluación) encontramos diferencias significativas en función a la Facultad de pertenencia, siendo los estudiantes de Educación quienes se definen con tendencia a la premorbidad de males cardiovasculares, siendo conveniente destacar que hablamos de tendencias y no de Tipos definidos.

c) En el Tipo 3, no se encuentran diferencias significativas en el momento 2; sí en cambio en la interacción del momento 1, donde la Facultad de pertenencia contribuyó inicialmente, básicamente en la de Educación. Al desvanecerse con el tiempo y al ubicar el momento 2, los promedios dentro de los límites del cuartil dos, muestran que los resultados obtenidos en el Tipo 1 no logran atenuar significativamente. Como se recordará los sujetos definidos como Tipo 3 están protegidos de la premorbidad oncológica y cardiovascular.

d) En el Tipo 4, saludable, no ubicamos diferencias significativas por facultades o por el sexo. Se encuentra que los estudiantes en general bordean el límite inferior del cuartil 
tres. Dicho resultado significa una contribución atenuante a los efectos tanto del tipo 1 como del tipo 2.

e) En el Tipo 5, no se registran diferencias significativas. Las puntuaciones promedio bordean los límites del cuartil 1, pudiendo interpretarse que el control de las funciones mediante la racionalización-siendo espúreamente mayor en los varones que las mujeresrevela que este tipo no es dominante en los grupos examinados, disminuyendo de esta manera la probabilidad de la definición de cuadros oncológicos y cardiovasculares.

f) En el tipo 6, se encuentra incrementos significativos en función a la Facultad de pertenencia, siendo los estudiantes de educación quienes presentan una mayor tendencia hacia rasgos psicopáticos, aún cuando las puntuaciones promedio no se ubican dentro del cuartil tres.

\section{Análisis de Contingencias:}

Con el propósito de establecer los cambios del tipo de personalidad del momento 1 al momento 2 los resultados se analizaron mediante el análisis de contingencia de Mc. Nemar donde cada sujeto actúa como su propio control, de forma tal que se puede observar si un sujeto se mantiene, cambia de lo negativo a lo positivo, o lo contrario. Así también es detectable si se mantiene sin diferencias al estado inicial. Nos interesa básicamente establecer si hay cambios significativos por ser un indicador de la premorbidad en aquellos casos donde los cambios van de lo positivo a lo negativo. Estos resultados se reproducen en el siguiente cuadro resumen, donde se podrán observar las frecuencias absolutas para cada dirección de cambio y el valor de la contingencia en cada uno de los seis tipos de personalidad evaluadas.

\section{Cuadro $\mathbf{N}^{\circ} 6$ \\ Análisis de contingencia de los cambios del tipo de personalidad del momento 1 al momento 2}

\begin{tabular}{|c|c|c|c|c|c|c|}
\hline \multirow[t]{2}{*}{ Tipo } & \multicolumn{3}{|c|}{ PSICOLOGÍA } & \multicolumn{3}{|c|}{ EDUCACIÓN } \\
\hline & $\begin{array}{l}\text { CAMBIO } \\
-\quad+\end{array}$ & $\begin{array}{r}\text { CAMBIO } \\
-\quad+\end{array}$ & $\mathbf{X}$ & $\begin{array}{r}\text { CAMBIO } \\
-\end{array}$ & $\begin{array}{l}\text { CAMBIO } \\
-\quad+\end{array}$ & $\mathbf{X}$ \\
\hline 1 & 6 & 15 & 3.05 & 6 & 17 & $4.35^{*}$ \\
\hline 2 & 5 & 18 & $6.26^{*}$ & 4 & 27 & 0.36 \\
\hline 3 & 7 & 13 & 1.25 & 4 & 8 & 0.75 \\
\hline 4 & 10 & 10 & 0.05 & 10 & 13 & 0.17 \\
\hline 5 & 7 & 13 & 1.25 & 9 & 2 & 0.19 \\
\hline 6 & 11 & 12 & 0.00 & 7 & 7 & 0.07 \\
\hline
\end{tabular}

* Indica cambio significativo al 0.05 de margen de error, cuyo valor crítico es 3.84

En los estudiantes de la Facultad de Psicología se encuentra un cambio significativo para bien en el tipo 2, lo que indica que estudiantes que en el momento uno se perfilaban como propensos a males cardiovasculares significativamente han cambiado sus reacciones interpersonales. En los estudiantes de Educación ha ocurrido algo similar en el tipo 1.

Los cambios que se pueden observar en todos los demás tipos no llegan a ser significativos, siendo conveniente señalar que el signo negativo del cuadro (-) indica puntuaciones menores en sus reacciones interpersonales y el positivo (+) indica lo contrario, es decir, presencia de indicadores patógenos.

Finalmente, es conveniente describir a los grupos examinados en función a la conservación del tipo de personalidad y cambios, sean positivos o negativos. Dichos datos han sido analizados en función a la Facultad de pertenencia y a continuación se presentan. 
Cuadro No. 7

Frecuencias absolutas y relativas de la Evolución de los tipos de Personalidad con Intervalos de 6 meses en 69 estudiantes del primer año de la Facultad de Psicología

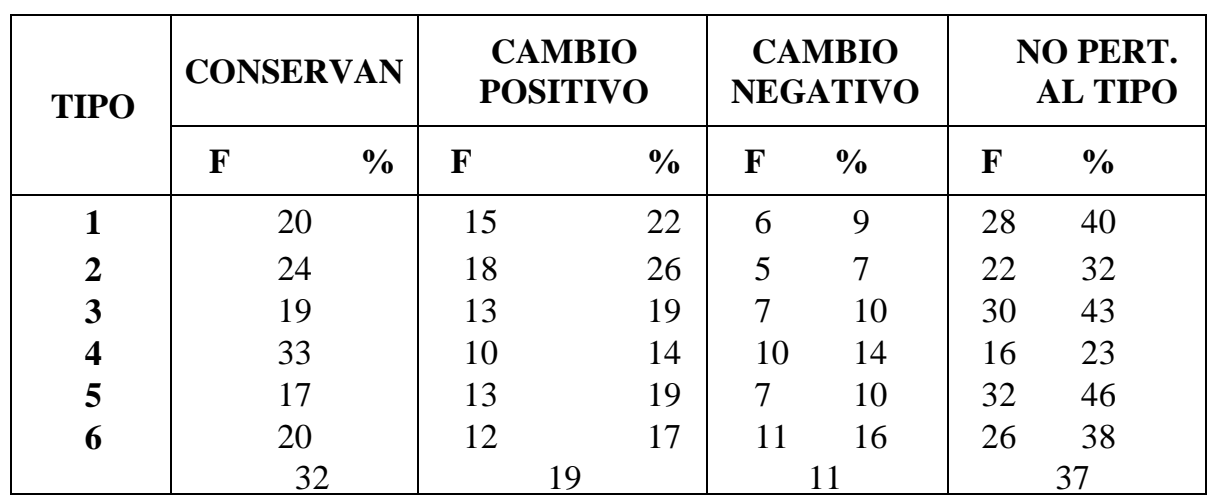

De la lectura del cuadro se desprende:

a) Que hay un $38 \%$ de examinados que se ubican como tipos definidos a la premorbidad oncológica. El $29 \%$ por conservarse en el tipo y un $9 \%$ por experimentar cambio negativo en el momento dos.

b) Hay un $42 \%$ de estudiantes de la Facultad de Psicología con premorbidad a males cardiovasculares.

c) Hay un $38 \%$ que presenta rasgos histéricos protegidos de posibles cuadros oncológicos y cardiovasculares.

d) Un $62 \%$ presenta personalidad saludable.

e) Un 35\% presenta características de control emocional mediante la racionalización, con propensión a males cardiovasculares y también oncológicos.

f) Un $40 \%$ muestra características psicopáticas, que podrían devenir en drogadicción y criminalidad.

Es importante señalar que, como cada examinado es caracterizado en función a seis tipos de reacciones interpersonales tendrá a su vez seis resultados, con la posibilidad de que en más de uno esté comprendido en el cuartil 3 (tipo definido). Así, la estimación de los porcentajes se ha realizado sobre el total posible de combinaciones de resultados, siendo su suma igual a 414 (o sea 6 resultados por cada uno de los 69 sujetos).

Cuadro No. 8

Frecuencias absolutas y relativas de la Evolución de los tipos de personalidad con intervalos de 6 meses en 72 estudiantes del 1er. año de Educación

\begin{tabular}{|c|c|c|c|c|c|c|c|c|}
\hline \multirow[t]{2}{*}{ TIPO } & \multicolumn{2}{|c|}{$\begin{array}{c}\text { CONSERVA } \\
\mathbf{N}\end{array}$} & \multicolumn{2}{|c|}{$\begin{array}{c}\text { CAMBIO } \\
\text { POSITIVO }\end{array}$} & \multicolumn{2}{|c|}{$\begin{array}{c}\text { CAMBIO } \\
\text { NEGATIVO }\end{array}$} & \multicolumn{2}{|c|}{$\begin{array}{l}\text { NO PERT. } \\
\text { AL TIPO }\end{array}$} \\
\hline & $\mathrm{F}$ & $\%$ & $\mathrm{~F}$ & $\%$ & $\mathrm{~F}$ & $\%$ & $\mathrm{~F}$ & $\%$ \\
\hline 1 & 21 & 29 & 17 & 24 & 6 & 8 & 28 & 39 \\
\hline 2 & 25 & 35 & 7 & 10 & 4 & 5 & 36 & 50 \\
\hline 3 & 32 & 44 & 8 & 11 & 4 & 5 & 28 & 39 \\
\hline 4 & 35 & 49 & 13 & 18 & 10 & 14 & 14 & 19 \\
\hline 5 & 22 & 30 & 12 & 17 & 9 & 12 & 29 & 40 \\
\hline \multirow[t]{2}{*}{6} & 23 & 32 & 7 & 10 & 7 & 10 & 35 & 48 \\
\hline & \multicolumn{2}{|c|}{36} & \multicolumn{2}{|c|}{15} & \multicolumn{2}{|c|}{9} & & 39 \\
\hline
\end{tabular}

Los sujetos que se definen con premorbidad a males oncológicos y cardiovasculares representan a 79 resultados, que vienen a ser el 19.08\%, al ubicarse en el cuartil tres de los 
tipos 1, 2 y 5; en tanto que la diferencia, es decir, 335 resultados representan el $80.92 \%$ de los resultados, que corresponden a reacciones interpersonales saludables.

De la lectura del cuadro se desprende:

a) Que hay un $37 \%$ de examinados que se ubican como tipos definidos a la premorbidad oncológica. El $29 \%$ por conservarse en el tipo y un $8 \%$ por experimentar cambio negativo en el momento dos.

b) Hay un $40 \%$ de estudiantes de la Facultad de Educación con premorbidad a males cardiovasculares.

e) Hay un $49 \%$ que presenta rasgos histéricos protegidos de posibles cuadros oncológicos y cardiovasculares.

f) Un $63 \%$ presenta personalidad saludable.

g) Un $42 \%$ presenta características de control emocional mediante la racionalización, con propensión a males cardiovasculares y también oncológicos.

1) Un $42 \%$ muestra características psicopáticas, que podrían devenir en drogadicción y criminalidad.

Es importante señalar que, como cada examinado es caracterizado en función a seis tipos de reacciones interpersonales tendrá a su vez seis resultados, con la posibilidad de que en más de uno esté comprendido en el cuartil 3 (tipo definido). Así, la estimación de los porcentajes se ha realizado sobre el total posible de 432 combinaciones de resultados, siendo su suma igual a (o sea 6 resultados por cada uno de los 72 sujetos).

Los sujetos que se definen con premorbidad a males oncológicos y cardiovasculares representan a 87 resultados, que vienen a ser el $20.14 \%$, al ubicarse en el cuartil tres de los tipos 1,2 y 5 ; en tanto que la diferencia, es decir, 347 resultados representan el $79.86 \%$ de los resultados, que corresponden a reacciones interpersonales saludables.

\section{DISCUSIÓN}

De los resultados hallados en la presente investigación se ha encontrado que la Facultad de pertenencia marca diferencias sólo en el tipo 2, que presentan premorbidad a males cardiovasculares, en el tipo 6, que se caracteriza por rasgos psicopáticos. En ambos casos los promedios son significativamente mayores en los estudiantes de la Facultad de Educación respecto de los estudiantes de la Facultad de Psicología.

Respecto al sexo, sólo en el tipo 1, de premorbidad oncológica, encontramos diferencias significativas puesto que los varones presentan promedios significativamente mayores que el grupo de las mujeres.

El hallazgo de estos resultados procede del uso del inventario de Reacciones Interpersonales de Grossarth-Maticek y Eysenck, cuyos resultados vienen siendo analizados los últimos 40 años, con confiabilidad alta, validez concurrente y de constructo demostradas y que en la presente investigación se revisó el contenido idiomático

y la con fiabilidad mediante la consistencia interna, resultado confirmatorio con lo hallado por los autores.

De esta manera los resultados obtenidos aún cuando no llegan al 25\% de estudiantes que presentan premorbidad oncológica, cardiovascular o psicopática, se hace necesario desarrollar mediante las oficinas de asesoría y bienestar del estudiante programas psicológicos correctivos sobre el control y manejo del estrés, así como también fortalecer los mecanismos de la autoestima por ser un elemento que contribuye positivamente al control emotivo, emocional y racional de los estados tensionales. Esto último es conveniente analizado en próximas investigaciones. 


\section{BIBLIOGRAFIA}

Baltrash, H., Stangell, W., \& Waltz, M. Cancer bajo el punto de vista de la Conducta Patrón C. Activas Nervosa Superior, 1988,30,18-30

Hooth-Kewley, S., \& Friedman, H. Predictores psicológicas de las enfermedades al corazón, una revisión cualitativa. Psychological Bulletin, 1987, 101.343-362.

Eysenck, H. J. La personalidad, el cáncer y enfermedades cardiovasculares: Un análisis causal. Personality and Individual Differences. Lexington, MA: Heath, 1986. pp. 17 - 88.

Eysenck, H. J. Ansiedad, inutilidad adquirida (aprendida) y el cáncer - una teoría causal. Joumal of Anxiety Disorders, 1987, I 87 - 104 (a)

Eysenck, H. J. La personalidad como predictor del cáncer y las enfermedades cardiovasculares y la aplicación de la terapia conductual en la profilaxis. The European Joumal of Psychiatry, 1987,1,24-41 (b)

Eysenck, H. J. Personlinchkeit. Stress und Krankheit: Eine kausale Theorie. In Bericht uber den 35 Kongress del Deutschen Gessellschaft fur Psychologie - Heidelberg, 1986. Gottingen: Verlag fur Psychologie. 1987. Vol. 2. Pp. 387 -401. (c)

Eysenck, H. J La personalidad, el stress y el cáncer, predicción y profilaxis. British Joumal of Medical Psychology, 1988,61,57 - 75 (a).

Eysenck, H. J. La importancia respectiva de la personalidad, el fumar cigarrillos y efectos de interacción para el génesis del cáncer y enfermedades a la coronaria. Personality and Individual Differences, $1988,9,453$ - 464.(b)

Eysenk, H. J Conducta Tipo A y enfermedades coronarias. La tercera etapa. Joumal of Social Behavior and Personality, 1990, 5.25 - 44.

Eysenck, J. J., \& Grossarth- Maticek, R. La prevención del cáncer y las enfermedades a la coronaria y la reducción en el costo del servicio de salud nacional Joumal of Social, Political and Economic Studies, 1989, $14,25-47$.

Friedman, H. S., \& Booth-Kewley, S. La personalidad, conducta Tipo A y enfermedad a la coronaria, el rol de la expresión emocional. Joumal of Personality and Social Psychology, 1987,53.783 - 792.

Grossarth-Maticek, R., Bastiaans, J, \& Kanazir, D. T. Factores psicosociales como fuentes predictivas de la mortalidad por cáncer, ataque al corazón y enfermedad isquémica del corazón. Un estudio prospectivo Yugoeslavo. Joumal of Psychosomatic Research, 1985,29, 167-176.

Grossarth-Maticek, R., \& Eysenck, H. J. Tiempo de supervivencia y porcentaje de linfocitos en las mujeres con cáncer mamario como función de la psicoterapia. Pshychological Reports, 1989, 65m 315 - 321.

Grossarth-Maticek, R., Eysenck, H. J, \& Verter, H. Tipo de personalidad, hábito de fumar y su interacción sobre los predictores del cáncer y enfermedades de la coronaria. Personality and Individual Differences, 1988,9,479 - 495.

Grosasarth-Maticek, R., Eysenck, H. J., Vetter, H., \& Frentzel-Byme, R. Estudio de intervención prospectiva Heidelberg. In J. Eylenbosch, N. Van Larebeke, \& A. M. Depoorter (Eds.), Primary prevention of cancel', New York: Raven, 1988. Pp. 129-212.

Grossarth-Maticek, R., Kanazir, D. T., Schmidt, P., \& Vetter H. Variables orgánicas y psicosociales como predictores del cáncer al pulmón, infarto cardiaco y apoplegia: algunos predictores diferenciales. Personality and Individual Differences. 1985,6,313 - 321.

Grossarth-Maticek, R., Kanazir, D. T., Vetter, H. \& Schmidt P. Factores psicosomáticos dentro del proceso de cancerogénesis. Psichoterapy and Psychosomatics,1983,40.191-210.

Ploeg, H. van der Kleijn, W. C., Mook, 1, Hnge, M. Van Pieters, A., \& Lewer, J. M. El racionalismo y el antiemocionalismo como un factor de riesgo productor de cáncer: diferenciación de concepto. Joumal of Psychomatic Research, 1989,33,217 - 225.

Roseman, R. H., Chesnay, M. A. La relación del patrón de conducta tipo A a la enfermedad coronaria. Activas Nervosa Superior, 1980, 22m 1-45. 